\title{
CANARDS IN ADVECTION-REACTION-DIFFUSION SYSTEMS IN ONE SPATIAL DIMENSION
}

\author{
KRISTEN HARLEY
}

(Received 17 April 2015; first published online 16 June 2015)

2010 Mathematics subject classification: primary 34E17; secondary 34A12, 34C37, 34E15, 35C07, $92 \mathrm{C} 17$.

Keywords and phrases: advection-reaction-diffusion systems, travelling wave solutions, geometric singular perturbation theory, canards.

We explore the existence of travelling wave solutions to coupled, advectionreaction-diffusion (ARD) equation systems in one spatial dimension that come from mathematical biology. The specific ARD systems of interest are those in which diffusion is small (relative to the other terms), such that the systems are singularly perturbed. By looking for travelling wave solutions, the ARD systems are reduced to singularly perturbed systems of first-order ordinary differential equations, which we analyse using geometric singular perturbation theory (GSPT) and canard theory. Travelling wave solutions are then represented by heteroclinic orbits in a multidimensional phase plane.

Singular perturbation problems possess an inherent separation of scales. Geometric singular perturbation theory enables us to separate these scales into independent, lower-dimensional subsystems, which are more amenable to analysis. We construct solutions to the full system as concatenations of components from each of the subsystems. The justification for this method is provided by GSPT, specifically Fenichel theory. Canard theory is required, in addition to standard GSPT, when there is a fold in a critical manifold, along which the system loses normal hyperbolicity and hence Fenichel theory no longer applies. Along this fold, canard points can exist that allow solution trajectories to pass through the fold curve, via the canard point. These trajectories are either canard solutions, which pass from the attracting to the repelling branch of the critical manifold, or faux canard solutions, which pass from the repelling to the attracting branch. For the ARD models under consideration in this thesis, the combination of the folded nature of the critical manifold and the existence

Thesis submitted to Queensland University of Technology in September 2014; degree approved on 5 December 2014; supervisors: Graeme Pettet and Peter van Heijster.

(c) 2015 Australian Mathematical Publishing Association Inc. 0004-9727/2015 \$16.00 
of canard points, specifically folded saddle canard points, is essential for the existence of travelling wave solutions with steep fronts, which become shocks in the singular, diffusion-free limit.

Our analysis focuses on three specific models from the mathematical biology literature: a haptotaxis-dominated model of malignant tumour growth [2], a model of chemotactically driven wound healing angiogenesis [3] and a version of the KellerSegel model for which explicit solutions are known [1].

Firstly, we prove the existence of travelling wave solutions for an extended, biologically more realistic version of the malignant tumour model in [2] with small, rather than zero, diffusion. In the singular limit of no diffusion, these solutions reduce to those that have previously been identified numerically and can be classified into three distinct types. As well as providing a first proof of existence of the three types of solutions, our results validate a modelling assumption that the effect of diffusion is negligible, which is not evident in the original analysis. Furthermore, we prove the existence of a fourth type of wave that is physically unrealistic due to a component with negative cell density and so was previously neglected.

Secondly, we prove the existence of a novel, shock-fronted travelling wave solution for the wound healing model in [3] assuming that two conjectures hold. The conjectures relate to the validity of numerically observed behaviour. A proof of existence of a previously identified smooth travelling wave solution is also provided. In addition, we generalise these results to broader parameter regimes and discuss the potential for more exotic solutions: for example, where a solution trajectory passes through multiple canard points. The analysis of this model is similar to that of the malignant tumour model. However, it is significantly more involved due to the addition of two model parameters that complicate the slow dynamics. For example, up to five canard points may exist along the fold in the critical manifold, which interact and change type as the parameters vary, rather than a single folded saddle canard point.

Finally, we investigate the existence of travelling wave solutions for a version of the Keller-Segel model for which explicit solutions are known in the diffusion-free limit. In this context, we also demonstrate the effects that small but unequal diffusion coefficients have on the results.

\section{References}

[1] D. L. Feltham and M. A. J. Chaplain, 'Travelling waves in a model of species migration', Appl. Math. Lett. 13(7) (2000), 67-73.

[2] A. J. Perumpanani, J. A. Sherratt, J. Norbury and H. M. Byrne, 'A two parameter family of travelling waves with a singular barrier arising from the modelling of extracellular matrix mediated cellular invasion', Physica D 126(3-4) (1999), 145-159.

[3] G. J. Pettet, D. L. S. McElwain and J. Norbury, 'Lotka-Volterra equations with chemotaxis: walls, barriers and travelling waves', IMA J. Math. Appl. Med. Biol. 17 (2000), 395-413.

\section{KRISTEN HARLEY, Mathematical Sciences School, Queensland University of Technology, Brisbane, Australia e-mail: kristen.e.harley@gmail.com}

